\title{
Electron Backscatter Diffraction in the SEM: A Tutorial
}

\author{
J. R. Michael
}

Materials Characterization Dept., Sandia National Laboratory, PO Box 5800, Albuquerque, NM 87109-0886

Electron backscatter diffraction (EBSD) has become a common technique used in the characterization of polycrystalline materials. A search of a published literature citation index (SciSearch) is shown in Fig. 1 and demonstrates the rapid growth in the use of EBSD. This tutorial will start with a description of how these patterns are generated within the SEM. The tutorial will then discuss the application of EBSD to phase identification and automated orientation mapping. Finally the topic of how to select a system for your laboratory will be discussed.

ESBD patterns are generated in the SEM by illuminating a highly tilted sample $\left(>45^{\circ}\right)$ with a stationary electron probe. The electrons are scattered beneath the sample surface to form a divergent source of radiation. Electrons which have lost very little energy and are backscattered may either be channeled or diffracted into flat cones of higher electron intensity which when imaged on a phosphor screen appear as pairs of parallel nearly straight lines. There are pairs of lines due to interactions with either the front or the back of the atomic planes. Figure 2 shows an EBSD pattern from CdTe collected with a slow-scan CCD camera at an accelerating voltage of $20 \mathrm{kV}$.

Orientation mapping using EBSD is accomplished by obtaining EBSD patterns at an array of points on a sample. At each pixel the EBSD pattern is collected and automatically indexed and the crystallographic orientation calculated. A map of the crystallographic orientation (texture) of the sample can then be formed. Figure 3 is an example of an orientation map of electrodeposited Ni after an annealing treatment. The map is color coded so that pixels with orientations close to $\langle 100\rangle$ are red and those close to $\langle 111\rangle$ are blue. The blue, more equiaxed grains that are near the left side of the image are new recrystallized grains. It is possible to follow the progress of recrystallization as a function of time and temperature from orientation maps.

The identification of crystalline phases is now routine using EBSD patterns. Phase identification usually involves a search of a suitable crystallographic database with the crystallographic information from EBSD combined with qualitative chemical information from EDS. Figure 4 is an example of the identification of a small particle that contained $\mathrm{Pb}$ as Plattnerite $\left(\mathrm{PbO}_{2}\right)$. EBSD and the chemistry of the particle allowed the identification to be accomplished.

EBSD in the SEM is a technique that can provide a vast amount of information about crystalline materials. Orientation mapping provides information about the orientation of polycrystalline materials and the relation of these orientations to significant microstructural features. Phase identification using EBSD is now routinely used in the SEM to identify materials by their crystallography and chemistry [1].

References

[1] This work was supported by the United States Department of Energy under contract DE-AC0494AL8500. Sandia is a multiprogram laboratory operated by Sandia Corporation, a Lockheed Martin Company, for the U. S. Department of Energy. 

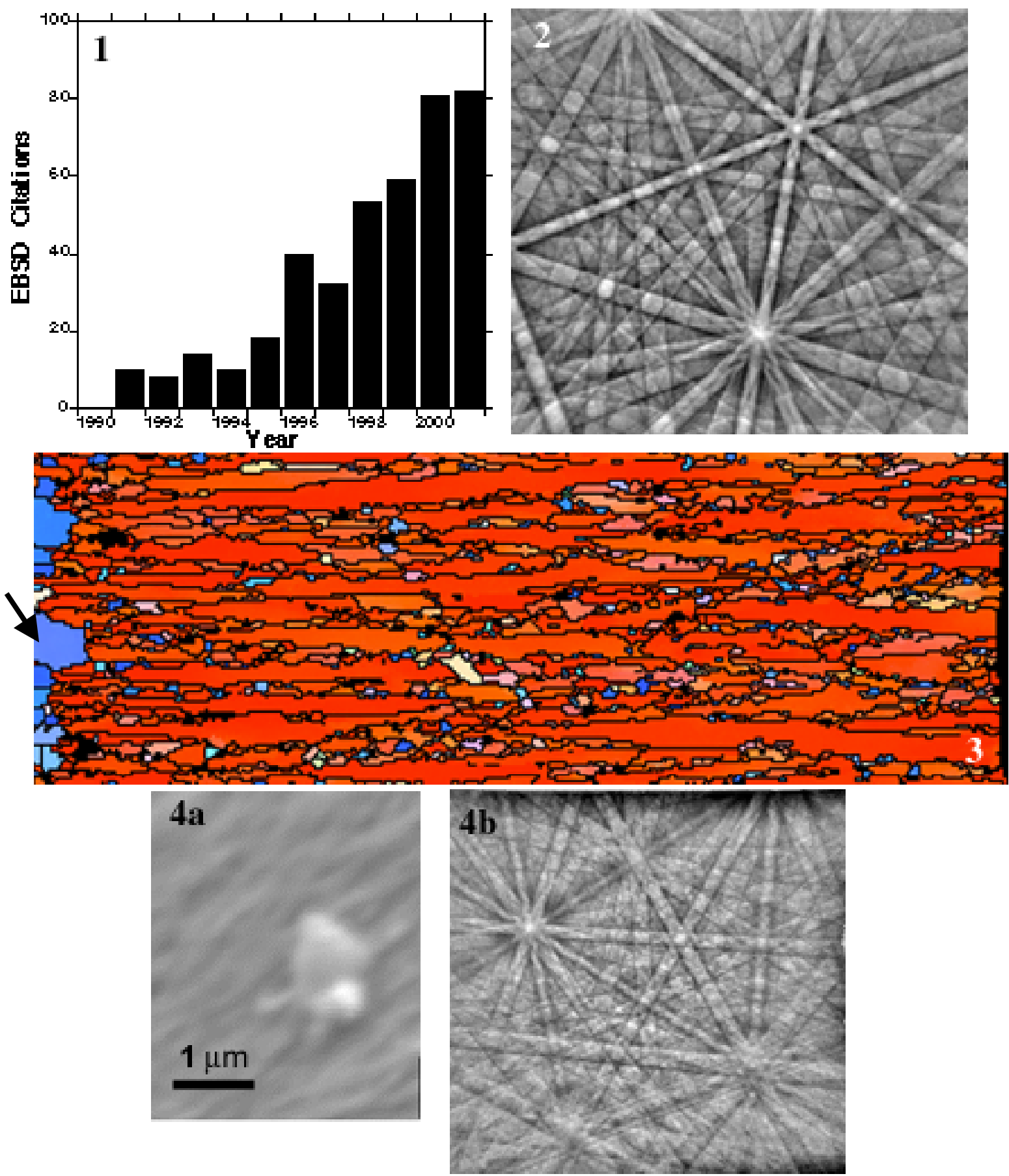

Fig. 1. Number of papers containing EBSD or EBSP published over the past 12 years.

Fig. 2. EBSD pattern of CdTe solar cell material obtained at $20 \mathrm{kV}$ and acquired using a slow-scan CCD-based camera.

Fig. 3. Orientation map of a cross section of electrodeposited $\mathrm{Ni}$ on $\mathrm{Cu}$. The large equiaxed recrystallized grains at the left of the image formed at the electrodeposit/substrate interface (one is indicated by an arrow). The full width of the image is $150 \mu \mathrm{m}$.

Fig. 4. Identification of small particle of $\mathrm{PbO}_{2}$. a) particle on bulk $\mathrm{C}$ substrate, b) EBSD pattern used to identify particle as Plattnerite $\left(\mathrm{PbO}_{2}\right)$. 